\title{
Decompositions of Continuity In Simply Extended Topological Spaces
}

\author{
S. Nagarani \\ Department of Mathematics, N.M.S.S.V.N. College, Madurai District-625 706, Tamil Nadu, India. \\ E-mail: nagadoss97@yahoo.in.
}

Article History: Received: 11 January 2021; Accepted: 27 February 2021; Published online: 5 April 2021

ABSTRACT. In this paper, we obtain some decompositions of continuity in simply extended topological spaces.

\section{INTRODUCTION}

Semi-open, preopen sets, $\alpha$-open, $\beta$-open sets or semi-preopen sets play an important role in the research of generalizations of continuity. By using these sets several authors introduced and studied various types of modifications of continuity in topological spaces. Semi-continuity, precontinuity, $\alpha$-continuity, $\beta$ continuity or semi- precontinuity and other forms. In [4-6,17] and [18], the following notions are introduced. $D(\tau, s)$-sets, $D(\tau, p)$ sets, $D(\alpha, s)$-sets, $D(\alpha, p)$ sets and $D(\tau, s)$-continuity, $D(\tau, p)$-continuity, $D(\alpha, s)$-continuity, $D(\alpha, p)$-continuity. By using these notions and the notions of semi-continuity, pre-continuity, $\alpha$ continuity, $\beta$-continuity some decompositions of continuity are obtained.

The notion of g-closed sets is introduced in [8]. The notion of g-continuity is introduced and studied in [2]. Recently, Murugalingam [10] introduced certain generalizations of g-closed sets in topological spaces.

In $[13,15]$ and other research articles, the authors introduced and investigated the notions of minimal structures, m-spaces, M-continuity and $\mathbf{M}^{*}$-continuity. The notion of $\mathbf{M}^{*}$-continuity is introduced in [9]. In [12] the author introduced the notions of $D\left(m_{1}, m_{2}\right)$-sets, where $m_{1}$ and $m_{2}$ are minimal structures on nonempty set $\mathrm{X}$, and obtain useful results concerning these sets. By using these results we obtain general decompositions of M-continuity. As immediate consequences, generalizations of the results established in $[3,5,6,17,18]$ are

0 AMSMathematics Subject Classification: 54A05, 54D10.

Key words and phrases. B-preclosed, B- $\alpha$-closed, B-b-closed, B- $\beta$-closed. obtained as new forms of continuity and weak forms of continuity in topological spaces.

In this paper, we obtain some decompositions of continuity in simply extended topological spaces.

\section{PRELIMINARIES}

Definition 2.1. Let $X$ be a non-empty set and Levine [7] defined $\tau(B)=\left\{O \cup\left(O^{0} \cap B\right): O, O^{0} \in \tau\right\}$ and called it simple extension of $\tau$ by $B$, where $B / \in \tau$. We call the pair $(X, \tau(B))$ a simply extended topological space (briefly SETS). The elements of $\tau(B)$ are called B-open sets and the complements of B-open sets are called B-closed sets. The B-closure of a subset $S$ of $X$, denoted by $B c l(S)$, is the intersection of $B$-closed sets of X including $S$. The Binterior of $S$, denoted by Bint $(S)$, is the union of $B$-open sets of $X$ contained in $S$.

Definition 2.2. [11] Let $(X, \tau(B))$ be a SETS and $A \subseteq X$. Then $A$ is said to be

(1) $B$-semiopen if $A \subseteq B \operatorname{Bcl}(\operatorname{Bint}(A))$;

(2) $B$-preopen if $A \subseteq B \operatorname{Bint}(B c l(A))$; (3) $B$ - $\alpha$-open if $A \subseteq B \operatorname{Bint}(B c l(B i n t(A)))$;

(4) $B$ - $\beta$-open if $A \subseteq B c l(\operatorname{Bint}(B c l(A)))$.

The complement of B-semiopen (resp. B-preopen, $B$ - $\alpha$-open, $B$ - $\beta$-open) is said to be B-semiclosed (resp. Bpreclosed, $B$ - $\alpha$-closed, $B$ - $\beta$-closed).

In this paper, let us denote by $\sigma(\tau(B))$ (or $\sigma)$ the class of all B-semiopen sets on $X$, by $\pi(\tau(B))$ (or $\pi)$ the class of all B-preopen sets on $X$, by $\alpha(\tau(B))$ (or $\alpha)$ the class of all $B$ - $\alpha$-open sets on $X$, by $\beta(\tau(B))($ or $\beta)$ the class of all $B$ $\beta$-open sets on $X$.

Lemma 2.3. [16] Let $\left(X, m_{X}\right)$ be an $m$-space and $m_{X}$ satisfy property B. Then for a subset $A$ of $X$, the following properties hold:

(1) $A \in m_{X}$ if and only if $\operatorname{mint}(A)=A$,

(2) $A$ is $m_{X}$-closed if and only if $m c l(A)=A$,

(3) $\operatorname{mint}(A) \in m_{X}$ and $\operatorname{mcl}(A)$ is $m_{X}$-closed.

Theorem 2.4. [12] Let $X$ be a nonempty set and $m_{1}, m_{2}, m_{3}$ minimal structures on $X$ such that $m_{1}$ has property B and $m_{1} \subset m_{2} \subset m_{3}$. Then $m_{1}=m_{2} \cap D\left(m_{1}, m_{3}\right)$.

Remark 2.5. [11]

(1) Every open set is B-open set.

(2) Every B-open set is B-preopen.

(3) Every B-open set is B-semi-open. 


\section{SIMPLE EXTENSION OF TOPOLOGIES}

Definition 3.1. $A$ subset $A$ of a simply extended topological space $(X, \tau(B))$ is said to be $B$-b-open if $A \subseteq$ $\operatorname{Bcl}(\operatorname{Bint}(A)) \cup \operatorname{Bint}(\operatorname{Bcl}(A))$.

The complement of $B$-b-open set is called $B$-b-closed.

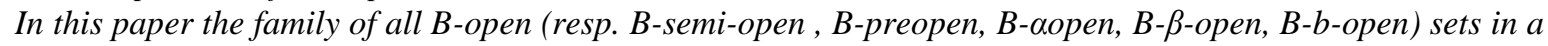
simply extended topological space $(X, \tau(B))$ is denoted by $B(X)$ (resp. $B S O(X) B P O(X), B \alpha(X), B \beta O(X), B b O(X)$ ). The following relations are well-known:

open $\quad-\rightarrow$ B-open $\rightarrow$ B-preopen

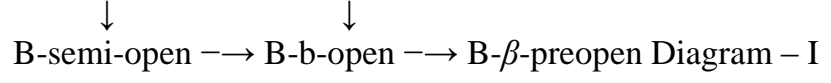

Example 3.2. Let $X=\{a, b, c, d, e\}, \tau=\{\varphi, X,\{a, b\},\{a, b, c, d\}\}$ and $B=\{c, d\}$.

Then $\tau(B)=\{\varphi, X,\{a, b\},\{c, d\},\{a, b, c, d\}\}$. We have

(1) $\{a\}$ is B-b-open set but not B-semi-open.

(2) $\{a, e\}$ is $B$ - $\beta$-open but not B-b-open.

Definition 3.3. In this chapter, the intersection of all B-semi-closed (resp. Bpreclosed, $B$ - $\alpha$-closed, $B$ - $b$-closed, $B$ $\beta$-closed) sets of $X$ containing $A$ is called the $B$-semi-closure (resp. B-preclosure, $B$ - $\alpha$-closure, $B$ - $b$-closure, $B$ - $\beta$ closure) of $A$ and is denoted by $B \operatorname{scl}(A)(\operatorname{resp} . \operatorname{Bpcl}(A), \operatorname{B\alpha cl}(A), \operatorname{Bbcl}(A), B \beta c l(A))$.

Definition 3.4. The union of all B-semi-open (resp. B-preopen, B $\alpha$-open, Bßopen, Bb-open) sets of $X$ contained in $A$ is called the B-semi-interior (resp. Bpreinterior, B $\alpha$-interior, B $\beta$-interior, Bb-interior ) of $A$ and is denoted by $B \operatorname{sint}(A)(\operatorname{resp} . B \operatorname{Bint}(A), \operatorname{B\alpha int}(A), B \beta \operatorname{Bint}(A), B \operatorname{bint}(A))$.

The family of all $B$-open (resp. B-semi-open, B-preopen, $B$ - $\alpha$-open, $B$ - $b$-open, $B \beta$-open) sets is denoted by $B(X)$ (resp. $B S O(X), B P O(X), B \alpha(X), B B O(X), B \beta(X)$ ).

We have the following implications

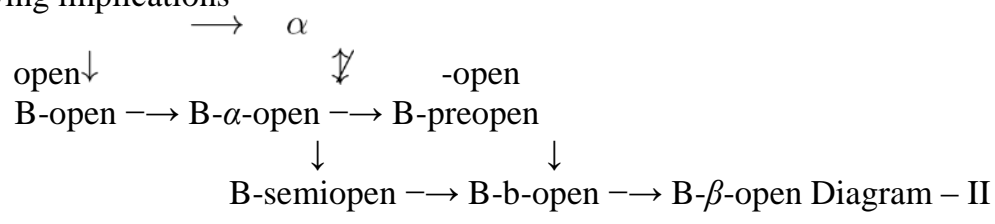

Example 3.5. Let $X=\{a, b, c\}, \tau=\{\varphi, X,\{a\}\}$ and $B=\{b\}$. Then $\tau(B)=\{\varphi, X,\{a\},\{b\},\{a, b\}\}$. Then $\{a, b\}$ is $\alpha$ open but not open.

Remark 3.6. $\alpha$-openness and $B$ - $\alpha$-openness are independent.

Example 3.7. Let $X=\{a, b, c\}, \tau=\{\varphi, X,\{a\}\}$ and $B=\{b\}$. Then $\tau(B)=\{\varphi, X,\{a\},\{b\}$, \{a,b\}\}. Then $\{a, c\}$ is $\alpha$ open but not $B$ - $\alpha$-open and $\{b\}$ is $B$ - $\alpha$-open but not $\alpha$-open.

Definition 3.8. Let $(X, \tau(B))$ be a simply extended topological space. A subset A of $X$ is said to be B-g-closed [1]

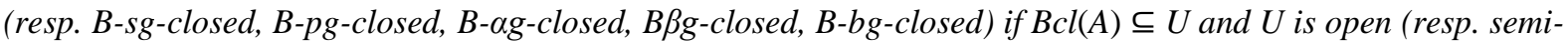
open, preopen, $\alpha$-open, $\beta$-open, b-open) in $(X, \tau)$. The complement of a B-g-closed (resp. B-sgclosed, B-pg-closed, $B$ - $\alpha$-closed, $B$ - $\beta g$-closed, B-bg-closed) set is a B-g-open (resp.

$B$-sg-open, B-pg-open, B- $\alpha$-open, B- $\beta g$-open, B-bg-open).

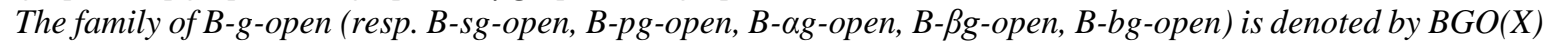
(resp. $B S G(X), B P G(X), B \alpha G(X), B \beta G(X), B b G(X)$ ).

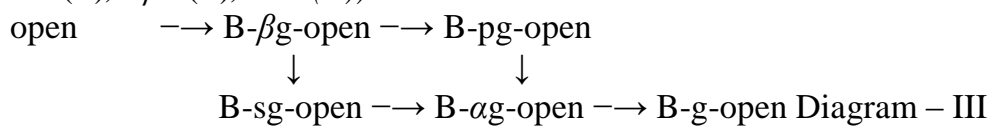

Example 3.9. Let $X=\{a, b, c\}, \tau=\{\varphi, X\}$ and $B=\{c\}$. Then $\tau(B)=\{\varphi, X$, $\{c\}\}$. Then

(1) $\{c\}$ is a B- $\beta$ g-open but not open.

(2) $\{a, b\}$ is B-sg-open but not B- $\beta g$-open.

(3) $\{a, b\}$ is B-ag-open but not B-pg-open

Example 3.10. Let $X=\{a, b, c\}, \tau=\{\varphi, X,\{a\}\}$ and $B=\{b\}$. Then $\tau(B)=\{\varphi, X,\{a\},\{b\},\{a, b\}\}$. Then $\{c\}$ is $B$ - $g$ open but not B-ag-open. 
Example 3.11. Let $X=\{a, b, c, d\}, \tau=\{\varphi, X,\{a, d\}\}$ and $B=\{c\}$. Then $\tau(B)$ $=\{\varphi, X,\{c\},\{a, d\},\{a, c, d\}\}$. Then

(1) $\{b\}$ is B-pg-open but not B- $\beta g$-open.

(2) $\{b\}$ is B-ag-open but not B-sg-open.

\section{MINIMAL STRUCTURES}

Remark 4.1. Let $(X, \tau)$ be a topological space and $(X, \tau(B))$ be a simply extended topological space. Then

(1) The families $\tau, S O(X), P O(X), \alpha(X), B O(X)$ and $\beta(X)$ are all m-structures on $X$.

(2) The families $B(X), B S O(X), B P O(X), B \alpha(X), B b O(X)$ and $B \beta O(X)$ are all also $m$-structures on $X$.

(3) $B G O(X), B S G(X), B P G(X), B \alpha G(X), B b G(X)$ and $B \beta G(X)$ are all also m-structures on $X$.

Remark 4.2. Let $(X, \tau)$ be a topological space and $(X, \tau(B))$ be a simply extended topological space and $A$ be a subset of $X$, then

(1) If $m_{X}=\tau($ resp. $S O(X), P O(X), \alpha(X), B O(X), \beta(X))$, then we have

(i) $\operatorname{mcl}(A)=\operatorname{cl}(A)(\operatorname{resp} . \operatorname{scl}(A), \operatorname{pcl}(A), \alpha c l(A), \operatorname{bcl}(A), \beta c l(A))$;

(ii) $\operatorname{mint}(A)=\operatorname{int}(A)(\operatorname{resp} . \operatorname{sint}(A), \operatorname{pint}(A), \operatorname{\alpha int}(A), \operatorname{bint}(A), \operatorname{\beta int}(A))$.

(2) If $m_{X}=B(X)($ resp. $B S O(X), B P O(X), B \alpha(X), B b O(X), B \beta O(X))$,

(i) $\operatorname{mcl}(A)=B c l(A)(\operatorname{resp} . B \operatorname{Bcl}(A), B p c l(A), B \alpha c l(A), B b c l(A), B \beta c l(A))$;

(ii) $\operatorname{mint}(A)=B \operatorname{int}(A)(\operatorname{resp} \cdot B \operatorname{sint}(A), B \operatorname{Bint}(A), B \alpha i n t(A), B \operatorname{bint}(A), B \beta \operatorname{int}(A))$.

(3) If $m_{X}=B G O(X)$ (resp. $B S G(X), B P G(X), B \alpha G(X), B b G(X), B \beta G(X)$ ), then we have

(i) $\operatorname{mcl}(A)=B \operatorname{Bgcl}(A)(\operatorname{resp} . B \operatorname{sgcl}(A), B \operatorname{Bgcl}(A), B \alpha \operatorname{gcl}(A), \operatorname{Bbgcl}(A), B \beta \operatorname{gcl}(A))$;

(ii) $\operatorname{mint}(A)=B \operatorname{gint}(A)(r e s p . B \operatorname{sint}(A), B \operatorname{pgint}(A), B \alpha \operatorname{gint}(A), B b \operatorname{gint}(A), B \beta \operatorname{gint}(A))$.

Remark 4.3. Let $(X, \tau(B))$ be a simply extended topological space. Then

(1) The families $S O(X), P O(X), \alpha(X), B O(X)$ and $\beta(X)$ are $m$-structures with property $\mathrm{B}$.

(2) The families $B(X), B S O(X), B P O(X), B \alpha(X), B b O(X)$ and $B \beta O(X)$ are $m$-structures with property $\mathrm{B}$.

(3) The families $B G O(X), B S G(X), B P G(X), B \alpha G(X), B b G(X), B \beta G(X))$ do not have property $\mathrm{B}$ in general. 5. $D\left(m_{1}, m_{2}\right)$-SETS

Definition 5.1. Let $(X, \tau(B))$ be a simply extended topological space, then we define the following:

(1) $D(\tau, B \alpha)=\{A \subset X: \operatorname{int}(A)=\operatorname{Baint}(A)\}$

(2) $D(B, B \alpha)=\{A \subset X: \operatorname{Bint}(A)=\operatorname{B\alpha int}(A)\}$,

(3) $D(B \alpha, B s)=\{A \subset X: B \operatorname{Bint}(A)=B \operatorname{sint}(A)\}$,

(4) $D(B s, B p)=\{A \subset X: B \operatorname{sint}(A)=B \operatorname{pint}(A)\}$.

Remark 5.2. Let $(X, \tau(B))$ be a simply extended topological space, then we have the following:

(1) $D(\tau, m)=\{A \subset X: \operatorname{int}(A)=\operatorname{mint}(A)\}$, where $m=B \beta g, B s g, B p g, B \alpha g$ or $B \beta g$.

(2) $D(\beta g, m)=\{A \subset X: \beta \operatorname{gint}(A)=\operatorname{mint}(A)\}$, where $m=B s g, B p g$, Bag or B-g.

(3) $D(B s g, m)=\{A \subset X: B \operatorname{sgint}(A)=\operatorname{mint}(A)\}$, where $m=B p g$, Bag or $B-g$.

(4) $D(B p g, m)=\{A \subset X: B \operatorname{pgint}(A)=\operatorname{mint}(A)\}$, where $m=B \alpha g$ or $B B g$.

(5) $D(B \alpha g, B g)=\{A \subset X: B \alpha \operatorname{gint}(A)=B \operatorname{gint}(A)\}$.

$D(\tau, B \alpha), D(B, B \alpha), D(B \alpha, B s)$ and $D(B s, B p)$ are defined in Definition 5.1.

Remark 5.3. Let $(X, \tau(B))$ be a simply extended topological space, then we have the following:

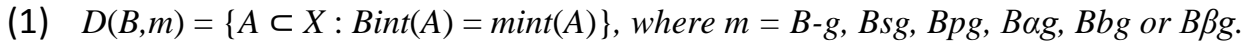

(2) $D(B, m)=\{A \subset X: \operatorname{Bint}(A)=\operatorname{mint}(A)\}$, where $m=B \alpha, B p, B s, B b$ or $B \beta$.

(3) $D(B \alpha, m)=\{A \subset X: B \alpha i n t(A)=\operatorname{mint}(A)\}$, where $m=B p$, Bs, Bb or $B \beta$.

(4) $D(B s, m)=\{A \subset X: B \operatorname{sint}(A)=\operatorname{mint}(A)\}$, where $m=B p$, Bb or $B \beta$.

(5) $D(B p, m)=\{A \subset X: B \operatorname{pint}(A)=\operatorname{mint}(A)\}$, where $m=B b$ or $B \beta$.

(6) $D(B b, B \beta)=\{A \subset X: \operatorname{Bbint}(A)=B \beta \operatorname{Bint}(A)\}$.

Theorem 5.4. Let $X$ be a nonempty set and $m_{1}, m_{2}$ minimal structures on $X$ such that $m_{1}$ has property $\mathrm{B}$ and $m_{1} \subset$ $m_{2}$. Then $m_{1}=m_{2} \cap D\left(m_{1}, m_{2}\right)$.

Proof. Let $V \in m_{1}$, then $V \in m_{2}$ and $V=m_{2} \operatorname{int}(V)$. Since $V \in m_{1}, V=m_{1} \operatorname{int}(V)$ and hence $V=m_{1} \operatorname{int}(V)=m_{2} \operatorname{int}(V$ ). Therefore, we have $V \in m_{2} \cap D\left(m_{1}, m_{2}\right)$ and hence $m_{1} \subset m_{2} \cap D\left(m_{1}, m_{2}\right)$. 
Conversely, suppose $V \in m_{2} \cap D\left(m_{1}, m_{2}\right)$. Since $V \in m_{2}, V=m_{2} \operatorname{int}(V)$. Since $V \in D\left(m_{1}, m_{2}\right), m_{1} \operatorname{int}(V)=m_{2} \operatorname{int}(V)$ and hence $V=m_{1} \operatorname{int}(V)$. Since $m_{1}$ has property B, by Lemma 2.3 we have $V \in m_{1}$ and $m_{2} \cap D\left(m_{1}, m_{2}\right) \subset m_{1}$.

Corollary 5.5. Let $(X, \tau(B))$ be a simply extended topological space. Then the following properties hold:

(1) $\tau(B)=B(X) \cap D(\tau, B)=B \alpha(X) \cap D(\tau, B \alpha)=B P O(X) \cap D(\tau, B P)$ $=B S O(X) \cap D(\tau, B S)=B B O(X) \cap D(\tau, B b)=B \beta(X) \cap D(\tau, B \beta)$,

(2) $B(X)=B \alpha(X) \cap D(B, B \alpha)=B P O(X) \cap D(B, B p)=B S O(X) \cap$ $D(B, B s)$ $=B B O(X) \cap D(B, B b)=B \beta(x) \cap D(B, B \beta)$,

(3) $B \alpha(X)=B P O(X) \cap D(B \alpha, B p)=B S O(X) \cap D(B \alpha, B s)=B B O(X) \cap D(B \alpha, B b)=B \beta(X) \cap D(B \alpha, B \beta)$,

(4) $B P O(X)=B B O(X) \cap D(B p, B b)=B \beta(x) \cap D(B p, B \beta)$, (5) $B S O(X)=B B O(X) \cap D(B s, B b)=B \beta(X) \cap$ $D(B s, B \beta)$,

(6) $B B O(X)=B \beta(X) \cap D(B b, B \beta)$

Proof. This is an immediate consequence of Theorem 5.4 and Diagram II.

Corollary 5.6. Let $(X, \tau(B))$ be a simply extended topological space. Then the following properties hold:

(1) $\tau(B)=B \beta G(X) \cap D(\tau(B), B \beta g)=B S G(X) \cap D(\tau(B), B s g)=B S G(X) \cap D(\tau(B), B p g)=B \alpha G(X) \cap$ $D(\tau(B), B \alpha g)=B G O(X) \cap$ $D(\tau(B), B g)$.

(2) $B S G(X)=B \alpha G(X) \cap D(B s g, B \alpha g)=B G O(X) \cap D(B s g, B g)$.

Proof. This is an immediate consequence of Theorem 5.4 and Diagram III.

Corollary 5.7. Let $(X, \tau(B))$ be a simply extended topological space. Then the following properties hold:

(1) $\tau=B(X) \cap D(\tau, m)$, where $m=B \alpha, B s, B p, B b$ or $B \beta$

$=B \alpha(X) \cap D(\tau, m)$, where $m=B s, B p, B b$ or $B \beta$

$=B S O(X) \cap D(\tau, B b)=B S O(X) \cap D(\tau, B \beta)=B P O(X) \cap D(\tau, B b)=B P O(X) \cap D(\tau, B \beta)=B B O(X) \cap$ $D(\tau, B \beta)$.

(2) $\quad B(X)=B \alpha(X) \cap D(B, m)$, where $m=B s, B p$, Bbor $B \beta=B P O(X) \cap D(B, B b)=B P O(X) \cap D(B, B \beta)=$ $B S O(X) \cap D(B, B b)=B S O(X) \cap D(B, B \beta)=B B O(X) \cap D(B, B \beta)$.

(3) $B \alpha(X)=B P O(X) \cap D(B \alpha, B b)=B P O(X) \cap D(B \alpha, B \beta)$

$=B S O(X) \cap D(B \alpha, B b)=B S O(X) \cap D(B \alpha, B \beta)=B B O(X) \cap D(B \alpha, B \beta)$.

(4) $B P O(X)=B B O(X) \cap D(B p, B \beta)$.

(5) $B S O(X)=B B O(X) \cap D(B s, B \beta)$.

(6) $B B O(X)=B \beta(X) \cap D(B b, B \beta)$.

Proof. This is an immediate consequence of Theorem 2.4 and Diagram II.

Corollary 5.8. Let $(X, \tau(B))$ be a simply extended topological space. Then the following properties hold:

(1) $\tau(B)=B \beta G(X) \cap D(\tau(B), m)$, wherem $=B s g, B p g, B \alpha g$ orBg $=B S G(X) \cap D(\tau(B), B \alpha g)=B S G(X) \cap D(\tau(B), B g)=B P G(X) \cap D(\tau(B), B \alpha g)=B \alpha G(X) \cap D(\tau(B), B g)=$ $B \alpha G(X) \cap D(\tau(B), B g)$.

(2) $B S G(X)=B \alpha G(X) \cap D(B s g, B g)$.

Proof. This is an immediate consequence of Theorem 2.4 and Diagram III.

\section{DECOMPOSITIONS OF CONTINUITY}

Remark 6.1. Let $(X, \tau(B))$ be a simply extended topological space and $m_{X}$ an m-structure on $X$.

(1) If $m_{X}=\tau(B)$ (resp. $B S O(X), B P O(X), B \alpha(X), B B O(X), B \beta(X)$ ), $m_{Y}=\sigma$ is a simply extended topology for $Y$ and $f:\left(X, m_{X}\right) \rightarrow\left(Y, m_{Y}\right)$ is

$M$-continuous, then $f$ is $B$-continuous (resp. B-semi-continuous, B-precontinuous, B $\alpha$-continuous, $B$ - $b$ continuous, $B$ - $\beta$-continuous $)$.

(2) If $m_{X}=B g(X)$ (resp. $\left.B S G(X), B P G(X), B \alpha G(X), B \beta G(X)\right), m_{Y}=\sigma$ is

a simply extended topology for $Y$ and $f:\left(X, m_{X}\right) \rightarrow\left(Y, m_{Y}\right)$ is $M^{*}$

continuous, then $f$ is Bg-continuous (resp. Bsg-continuous, B-pg-continuous,

Bag-continuous,

Bßg-continuous).

Definition 6.2. Let $X$ be a nonempty set and $m_{1}, m_{2}$ two minimal structures on $X$. 
A function $f:\left(X, D\left(m_{1}, m_{2}\right)\right) \rightarrow\left(Y, m_{Y}\right)$ is said to be $D\left(m_{1}, m_{2}\right)$-continuous iff is $M^{*}$-continuous, equivalently if the inverse image of each $m_{Y}$-open set of $Y$ is a $D\left(m_{1}, m_{2}\right)$-set of $X$.

Theorem 6.3. Let $X$ be a nonempty set and $m_{1}, m_{2}$ minimal structures on $X$ such that $m_{1}$ has property $\mathrm{B}$ and $m_{1} \subset$ $m_{2}$. Then a function $f:\left(X, m_{1}\right) \rightarrow\left(Y, m_{Y}\right)$ is

$M$-continuous if and only if

(1) $f:\left(X, m_{2}\right) \rightarrow\left(Y, m_{Y}\right)$ is $M^{*}$-continuous and

(2) $f:\left(X, D\left(m_{1}, m_{2}\right)\right) \rightarrow\left(Y, m_{Y}\right)$ is $D\left(m_{1}, m_{2}\right)$-continuous.

Proof. The proof follows immediately from Theorem 5.4.

Corollary 6.4. (1) For a function $f:(X, \tau(B)) \rightarrow(Y, \sigma(B))$, the following are equivalent:

(a) $f$ is continuous;

(b) fis B-continuous and $D(\tau, \tau(B))$-continuous;

(c) fis B $\alpha$-continuous and $D(\tau, B \alpha)$-continuous;

(d) fis Bp-continuous and $D(\tau, B p)$-continuous;

(e) $f$ is Bs-continuous and $D(\tau, B s)$-continuous; (f) $f$ is Bb-continuous and $D(\tau, B b)$-continuous;

(g) $f$ is $B \beta$-continuous and $D(\tau, B \beta)$-continuous.

(2) For a function $f:(X, \tau(B)) \rightarrow(Y, \sigma(B))$, the following are equivalent: (a) $f$ is $B$-continuous;
(b) $f$ is $B \alpha$-continuous and $D(B, B \alpha)$-continuous;
(c) fis Bp-continuous and $D(B, B p)$-continuous;
(d) fis Bs-continuous and $D(B, B s)$-continuous;
(e) $f$ is Bb-continuous and $D(B, B b)$-continuous;
(f) $\quad f$ is $B \beta$-continuous and $D(B, B \beta)$-continuous.

Proof. This is an immediate consequence of Corollary 5.5 and Theorem 6.3.

Remark 6.5. By Corollary 5.5(3)-(6) and Theorem 6.3, we can obtain several decompositions of B $\alpha$-continuity, Bp-continuity, Bs-continuity and Bb-continuity.

Corollary 6.6. (1) For a function $f:(X, \tau(B)) \rightarrow(Y, \sigma(B))$, the following are equivalent:

(a) $f$ is continuous;

(b) $f$ is B $\beta g$-continuous and $D(\tau, B \beta g)$-continuous;

(c) $f$ is Bsg-continuous and D( $\tau$,Bsg)-continuous;

(d) $f$ is Bpg-continuous and D( $\tau$,Bpg)-continuous;

(e) $f$ is Bag-continuous and D( $\tau, B \alpha g)$-continuous; (f) $f$ is Bg-continuous and $D(\tau, B g)$-continuous.

(2) For a function $f:(X, \tau(B)) \rightarrow(Y, \sigma(B))$, the following are equivalent: (a) $f$ is Bsg-continuous;

(b) fis Bag-continuous and D(Bsg,Bag)-continuous; (c) fis Bg-continuous and D(Bsg, Bg)-continuous. Proof. The proof follows immediately from Theorem 6.3 and Corollary 5.6.

Theorem 6.7. Let $X$ be a nonempty set and $m_{1}, m_{2}, m_{3}$ minimal structures on $X$ such that $m_{1}$ has property $\mathrm{B}$ and $m_{1}$ $\subset m_{2} \subset m_{3}$. Then a function $f:\left(X, m_{1}\right) \rightarrow\left(Y, m_{Y}\right)$ is M-continuous if and only if

(1) $f:\left(X, m_{2}\right) \rightarrow\left(Y, m_{Y}\right)$ is $M^{*}$-continuous and

(2) $f:\left(X, D\left(m_{1}, m_{3}\right)\right) \rightarrow\left(Y, m_{Y}\right)$ is $D\left(m_{1}, m_{3}\right)$-continuous.

Proof. The proof follows immediately from Theorem 2.4 .

Corollary 6.8. (1) For a function $f:(X, \tau(B)) \rightarrow(Y, \sigma(B))$, the following are equivalent:

(a) fis continuous;

(b) $f$ is B-continuous and $D(\tau, m)$-continuous, where $m=B \alpha, B s, B p$, $B b$ or $B \beta$;

(c) $f$ is $B \alpha$-continuous and $D(\tau, m)$-continuous, where $m=B s, B p, B b$ or $B \beta$;

(d) $f$ is Bs-continuous and $D(\tau, m)$-continuous, where $m=B b$ or $B \beta$; (e) f is Bp-continuous and $D(\tau, m)$ continuous, where $m=B b$ or $B \beta$; (f) $f$ is Bb-continuous and $D(\tau, B \beta)$-continuous.

(2) For a function $f:(X, \tau(B)) \rightarrow(Y, \sigma(B))$, the following are equivalent: (a) $f$ is B-continuous;

(b) $f$ is B $\alpha$-continuous and $D(\tau(B), m)$-continuous, where $m=B s, B p$, $B b$ or $B \beta$;

(c) $\quad$ is Bp-continuous and $D(\tau(B), m)$-continuous, where $m=B b$ or $B \beta$;

(d) $f$ is Bs-continuous and $D(\tau(B), m)$-continuous, where $m=B b$ or $B \beta$; (e) $f$ is Bb-continuous and $D(\tau(B), B \beta)$-continuous.

Proof. This is an immediate consequence of Corollary 5.7 and Theorem 6.7. 
Remark 6.9. By Corollary 5.7 and Theorem 6.7, we can obtain several decompositions of B $\alpha$-continuity, Bpcontinuity and Bs-continuity.

Corollary 6.10. (1) For a function $f:(X, \tau(B)) \rightarrow(Y, \sigma(B))$, the following are equivalent:

(a) fis continuous;

(b) $f$ is B Bg-continuous and $D(\tau, m)$-continuous, where $m=B \alpha g$ or $B g$

(c) $f$ is Bsg-continuous and $D(\tau, m)$-continuous, where $m=B a g$ or Bg; (d) $f$ is Bpg-continuous and $D(\tau, m)$-continuous, where $m=B \alpha g$ or Bg; (e) $f$ is Bag-continuous and $D(\tau, B g)$-continuous.

(2) A function $f:(X, \tau(B)) \rightarrow(Y, \sigma(B))$ is sg-continuous if and only if $f$ is Bag-continuous and $D(B s g, B g)$ continuous.

Proof. The proof follows immediately from Theorem 6.7 and Corollary 6.8.

\section{REFERENCES}

1. M. E. Abd El-Monsef, A. M. Kozae and R. A. Abu-Gdairi, New approaches for generalized continuous functions, Int. Journal of Math. Analysis, 4(27)(2010), 1329-1339.

2. K. Balachandran, P. Sundaram and H. Maki, On generalized continuous maps in topological spaces, Mem. Fac. Sci. Univ. Ser.A. Math., 12(1991), 5-13.

3. J. Dontchev and M. Przemski, On the various decompositions of some weakly continuous functions, Acta Math. Hungar., 71(1996), 109-120.

4. N. El-Deeb, I. A. Hasanein, A. S. Mashhour and T. Noiri, On p-regular spaces, Bull. Math.

5. Soc. Sci. Math. R. S. Roumanie, 27(75)(1983), 311-315.

6. M. Ganster, F. Gressl and I. L. Reilly, On decomposition of continuity, Lectures notes in Pure and Appl. Math., 134(1991), 67-72.

7. M. Ganster, F. Gressl and I. L. Reilly, Another decomposition of continuity, Annal New York Acad. Sci., 704(1993), 135-141.

8. N. Levine, Simple extension of topologies, Amer. Math. Monthly, 71(1964), 22-25.

9. N. Levine, Generalized closed sets in topology, Rend. Circ. Math. Palermo, (2), 19(1970), 89-96.

10. W. K. Min, $\mathbf{M}^{*}$-continuity and product minimal structures (submitted).

11. M. Murugalingam, A study of semi generalized topology, Ph.D Thesis, Manonmaniam Sundaranar University, Tirunelveli,Tamilnadu, 2005.

12. M. Murugalingam, O. Ravi and S. Nagarani, new Generalized Continuous Functions, International Journal of Mathematics and its Applications., (3), 3(2015), 55-62.

13. T. Noiri and V. Popa, On decompotions of continuity in topological spaces, Acta Math.

14. Hungar., 128(1-2)(2010), 175-189.

15. V. Popa and T. Noiri, On M-continuous functions, Anal. Univ. Dundrea de jos âAIJGalati, Ser. Mat Fiz Mec Teor., (2), 18(23)(2000), 31-41.

16. V. Popa and T. Noiri, A unified theory of weak continuity for functions, Rend. Circ. Mat.

17. Palermo, (2)51(2002), 439-464.

18. V. Popa and T. Noiri, On the definitions of some generalized forms of continuity under minimal conditions, Mem Fac. Sci. Kochi Univ. Ser. A Math., 22(2001), 31-41

19. V. Popa and T. Noiri, A unified theory of weak continuity for functions, Rend. Circ. Mat.

20. Palermo, (2)51(2002), 439-464.

21. M. Przemski, A decomposition of continuity, Commentationes Math., 33(1993), 153-157.

22. M. Przemski, A decomposition of contiuity and $\alpha$-continuity, Acta Math. Hungar., 61(1993), 93-98. 